\title{
Lipofection with Synthetic mRNA as a Simple Method for T-Cell Immunomonitoring
}

\author{
Natalia Teresa Jarzebska ${ }^{1,2} \oplus$, Julia Frei ${ }^{1,3}$, Severin Lauchli ${ }^{1,3}$, Lars E. French ${ }^{4,5}$, Emmanuella Guenova ${ }^{1,3,6}$, \\ Cécile Gouttefangeas ${ }^{7}\left(\mathbb{0}\right.$, Thomas M. Kündig ${ }^{1,3}{ }^{(D}$, Mark Mellett ${ }^{1,3}$ and Steve Pascolo ${ }^{1,3, *(1)}$
}

1 Department of Dermatology, University Hospital Zürich (USZ), University of Zürich (UZH), Raemistrasse 100, 8091 Zürich, Switzerland; NataliaTeresa.Jarzebska@usz.ch (N.T.J.); Julia.Frei@usz.ch (J.F.); Severin.Laeuchli@usz.ch (S.L.); emmanuella.guenova@unil.ch (E.G.); Thomas.Kuendig@usz.ch (T.M.K.); Mark.Mellett@usz.ch (M.M.)

2 Faculty of Science, University of Zürich, 8006 Zürich, Switzerland

3 Faculty of Medicine, University of Zürich, 8006 Zürich, Switzerland

4 Department of Dermatology and Allergy, University Hospital, LMU Munich, 80539 Munich, Germany; Lars.French@med.uni-muenchen.de

5 Dr. Philip Frost, Department of Dermatology and Cutaneous Surgery, University of Miami Miller School of Medicine, Miami, FL 33146, USA

6 Department of Dermatology and Venereology, Faculty of Biology and Medicine, Lausanne University Hospital (CHUV), University of Lausanne, 1000 Lausanne, Switzerland

7 Department of Immunology, University of Tübingen, 72074 Tübingen, Germany; Cecile.Gouttefangeas@uni-tuebingen.de

* Correspondence: Steve.Pascolo@usz.ch

check for updates

Citation: Jarzebska, N.T.; Frei, J.; Lauchli, S.; French, L.E.; Guenova, E.; Gouttefangeas, C.; Kündig, T.M.; Mellett, M.; Pascolo, S. Lipofection with Synthetic mRNA as a Simple Method for T-Cell Immunomonitoring. Viruses 2021, 13, 1232. https:// doi.org/10.3390/v13071232

Academic Editors: Ben van der Zeijst and Peter Rottier

Received: 7 May 2021

Accepted: 16 June 2021

Published: 25 June 2021

Publisher's Note: MDPI stays neutral with regard to jurisdictional claims in published maps and institutional affiliations.

Copyright: (C) 2021 by the authors Licensee MDPI, Basel, Switzerland. This article is an open access article distributed under the terms and conditions of the Creative Commons Attribution (CC BY) license (https:/ / creativecommons.org/licenses/by/ $4.0 /)$.

\begin{abstract}
The quantification of T-cell immune responses is crucial for the monitoring of natural and treatment-induced immunity, as well as for the validation of new immunotherapeutic approaches. The present study presents a simple method based on lipofection of synthetic mRNA in mononuclear cells as a method to determine in vitro T-cell responses. We compared several commercially available transfection reagents for their potential to transfect mRNA into human peripheral blood mononuclear cells and murine splenocytes. We also investigated the impact of RNA modifications in improving this method. Our results demonstrate that antigen-specific T-cell immunomonitoring can be easily and quickly performed by simple lipofection of antigen-coding mRNA in complex immune cell populations. Thus, our work discloses a convenient solution for the in vitro monitoring of natural or therapy-induced T-cell immune responses.
\end{abstract}

Keywords: transfection; ivt mRNA; immunomonitoring; lipofection; TLR7/8; T-cells

\section{Introduction}

The intricate complexity of the human immune system makes it challenging to analyze its responses to infection, disease, injury, or medical intervention. Immunomonitoring of T-cell responses provides information on the nature and state of immune reactions and is required to assess the efficacy of a medical treatment or to predict its effects [1]. Phenotyping of immune cell populations aids in the elucidation of the cellular mechanisms underlying newly developed immunotherapeutic approaches. It can also identify the presence of cellular and molecular signatures that categorize patients into distinct risk groups and/or help to predict clinical responses to therapy [2].

For the purpose of T-cell immunomonitoring, autologous target cells expressing epitopes of interest are usually required [3]. Defined epitopes can be introduced into immune cells through various methods, including overlapping peptides [4], viral vectors [5], or via the transfection of plasmid DNA or synthetic in vitro transcribed mRNA (ivt mRNA) coding the epitope or protein of interest [6,7]. 
Among these methods, mRNA is the most promising, as mRNA is easy to produce, and can encode full-length antigens or epitopes either in their wild-type form or in chimeric proteins for specific delivery to antigen presentation compartments [8-10].

The most popular strategy for immune response monitoring includes in vitro transfection by electroporation of antigen-presenting cells (APCs), such as dendritic cells (DCs), or of peripheral blood mononuclear cells (PBMCs) [3,10,11].

Such methodology based on electroporation is cumbersome and time consuming and is therefore not adequate for high-throughput T-cell immunomonitoring assays.

To attain a faster, easier, and more robust method for T-cell immunomonitoring, we explored whether freshly-isolated PBMCs could be transfected with mRNA using commercially available transfection reagents, including lipoplexes and polyplexes, to induce mRNA-coded epitope-specific immune responses. We aimed to define simple conditions to induce an immune response using mRNA in nanoparticles. This approach is an easy and straightforward method that can be implemented in high-throughput experiments.

\section{Materials and Methods}

\subsection{Messenger RNA Preparation}

Firefly Luciferase-coding mRNAs were produced using in vitro transcription at the "ivt mRNA production and formulation platform" in Zürich (http:/ / www.cancer.uzh.ch/ en/Research/mRNAPlatform.html, accessed on 26 January 2021). The $5^{\prime}$ end consisted of a CleanCap ${ }^{\mathrm{TM}}$ (Trilink, San Diego, CA, USA) followed by an eIF4G aptamer as the $5^{\prime}$ untranslated region [12] (that, as we could previously show, enhances translation of both unmodified and pseudouridine-modified ivt mRNA) and by a codon-optimized firefly luciferase open reading frame. The $3^{\prime}$ end consisted of a tandem repeat of the mouse beta globin $3^{\prime}$ UTR and a poly-A tail [13]. The transcription of mRNA was performed in the presence of the four canonical bases (A, C, G, and U) to obtain immuno-stimulatory RNA and in the presence of pseudouridine instead of uridine to obtain immuno-silent mRNA. RNA was diluted in RNase-free water, and the concentration (after quantification using a Nanodrop) was adjusted to $1 \mathrm{mg} / \mathrm{mL}$. The quality and integrity of ivt mRNAs were checked using agarose gel electrophoresis. The mRNAs were stored at $-20{ }^{\circ} \mathrm{C}$.

\subsection{Cell Culture and Luciferase Experiments}

Human embryonic kidney (HEK) 293 cells, murine colon carcinoma CT26 cells, and murine melanoma B16-F10 cells were maintained in RPMI medium (ThermoFisher Scientific, Waltham, MA, USA) containing 10\% fetal calf serum (FCS), $200 \mathrm{mM} 1$ glutamine (Gibco, Waltham, MA, USA), and $0.2 \%$ antimicrobial reagent Normocin (InvivoGen San Diego, CA, USA), named complete medium in the following. The following mRNA lipofection agents were used: Lipofectamine Messenger Max (Invitrogen, Waltham, MA, USA), RiboJuice mRNA transfection kit and RiboJuice siRNA transfection kit (Merck Millipore Sigma Aldrich St. Louis, MO, USA), mRNA-Fect (RJH Biosciences Edmonton, AB, Canada), Screenfect (ScreenFect Transfections, Eggenstein-Leopoldshafen, Germany), and JetMessenger (Polyplus Transfection, Illkirch-Graffenstaden, France). For luciferase experiments, transfection of the above-mentioned cell lines was performed with 100,000 cells per well in 200 microliters of complete medium. mRNA carriers were prepared according to manufacturer protocols and added to plated cells to obtain a final mRNA concentration of $1 \mu \mathrm{g} / \mathrm{mL}$, and incubated with cells for the indicated time. Luciferase activity was recorded one day after transfection by adding 25 microlitres of Bright-Glo luciferase assay solution (Promega, Madison, WI, USA) and measuring the activity using the GloMax Discover and Explorer Detection System equipment (Promega, Madison, WI, USA).

\subsection{Measuring the Stimulation of the Innate Immune Response}

Blood samples were obtained from healthy donors. Spleens were obtained from BALB/c or C57BL/ 6 mice housed with ethical approval from the Cantonal Veterinary Office of Zürich, Switzerland (license ZH215/17). Spleens were dilacerated and splenocytes 
were resuspended in $10 \mathrm{~mL}$ of complete medium. For both human and mouse cells, mononuclear cells were isolated using the Ficoll-Paque ${ }^{\mathrm{TM}}$ Plus (GE Healthcare Life Sciences, Marlborough, MA, USA) method. A total number of one million cells per well were plated on 96-well plates and incubated overnight with appropriate RNA carrier containing $200 \mathrm{ng}$ of RNA per well (200 $\mu \mathrm{L}$ cultures). Protamine RNA particles were prepared as described previously $[14,15]$. The next day, supernatants were taken, and IFN $\alpha$ concentrations were measured via ELISA by following the manufacturer's protocol (Human IFN $\alpha$ pan ELISA development kit, MABTECH, ELISA MAX Standard Set Mouse IFN $\alpha$, Biolegend, San Diego, CA, USA). The absorbance was measured at $450 \mathrm{~nm}$ with an ELISA reader (GloMax Discover and Explorer Detection System equipment, Promega, Madison, WI, USA) and cytokine concentrations were calculated according to a standard curve.

\subsection{Measuring the Stimulation of Adaptive Immunity in Murine Cells}

C57BL/6-Tg(TcraTcrb)1100Mjb (22) mice, also referred to as OT1 mice, were a generous gift from Pål Johansen (University Hospital Zurich). Spleen mononuclear cells from OT1 mice were isolated using the Ficoll-Paque ${ }^{\mathrm{TM}}$ Plus (GE Healthcare Life Sciences, Marlborough, MA) method. A total number of 200,000 cells per well $(200 \mu \mathrm{L}$ culture $)$ were plated on 96-well plates and incubated overnight with appropriate mRNA carrier containing $200 \mathrm{ng}$ of immunostimulating mRNA per well. The next day, supernatants were taken, and interleukin-2 (IL-2) concentration was measured via ELISA, as per the manufacturer's protocol (ELISA MAX Standard Set Mouse IL-2, Biolegend, San Diego, CA, USA). The absorbance was measured at $450 \mathrm{~nm}$ with an ELISA plate reader (GloMax Discover and Explorer Detection System equipment, Promega, Madison, WI, USA).

\subsection{Measuring the Stimulation of Adaptive Immunity in Human Cells}

Blood samples were obtained from healthy, HLA-A2-positive donors. Mononuclear cells were isolated using the Ficoll-Paque ${ }^{\mathrm{TM}}$ Plus (GE Healthcare Life Sciences, Marlborough, MA, USA) method. A total number of $1 \times 10^{6}$ cells per well were plated on 24-well plates and incubated with the appropriate mRNA carrier containing $200 \mathrm{ng}$ of each indicated mRNA per well ( $1 \mathrm{~mL}$ cultures). Starting day 4 , every other day of the culture, $10 \mathrm{U} / \mathrm{mL}$ IL-2 (R\&D Systems, Minneapolis, MN, USA) was added to the cells. Cells were cultured for 12 days. At day 12, cells were stained with phycoerythrin (PE)-HLA-A*0201 tetramers containing the conserved immunodominant HLA-A*0201 epitope from influenza matrix M1 [16], at a final concentration of $5 \mu \mathrm{g} / \mathrm{mL}$ in PBS (The Tetramer Shop, Kongens Lyngby, Denmark) during $15 \mathrm{~min}$ at room temperature. Then, the cells were stained with a fluorescein isothiocyanate (FITC)-conjugated anti-CD3 antibody and a peridininchlorophyll-protein complex (PerCP)-conjugated anti-CD4 antibody (Becton Dickinson, Heidelberg, Germany) in a PBS buffer supplemented with $0.5 \%$ bovine serum albumin and $2 \mathrm{mM}$ EDTA for $30 \mathrm{~min}$ at $4{ }^{\circ} \mathrm{C}$. The cells were washed twice with PBS before being fixed with $1 \%$ formaldehyde and analyzed by flow cytometry (LSRForstessa, BD Biosciences, New Jersey, NJ, USA).

\section{Results}

\subsection{Screening of an Optimal Transfection Agent for Primary Immune Cells}

We tested six different commercially available transfection agents (Table 1), four lipoplexes (MessengerMax, RiboJuice siRNA reagent, RiboJuice miRNA reagent, and Screenfect) and two polyplexes (mRNA-Fect and JetMessenger), for their capacity to transfect murine splenocytes and human PBMCs (in addition to both human and murine cancer cell lines). The transfection proficiency was determined by measuring luciferase activity $24 \mathrm{~h}$ post luciferase-coding ivt-mRNA lipofection, upon the addition of luciferin substrat. 
Table 1. Summary of the properties of transfection agents used in this paper.

\begin{tabular}{|c|c|c|c|c|c|c|}
\hline \multirow[b]{2}{*}{ Table } & \multirow[b]{2}{*}{ Compound } & \multicolumn{5}{|c|}{ Transfection Efficiency } \\
\hline & & HEK & СТ26 & B16F10 & $\begin{array}{c}\text { Murine } \\
\text { Splenocytes }\end{array}$ & hPBMCs \\
\hline Messenger Max & Liposome & ++ & ++ & + & ++ & ++ \\
\hline RiboJuice mRNA & cationic polymer/lipid mixture & +++ & +++ & +++ & +++ & +++ \\
\hline RiboJuice siRNA & cationic polymer/lipid mixture & + & $+1-$ & - & - & - \\
\hline mRNA-Fect & amphiphilic polymer & + & ++ & + & + & + \\
\hline Screenfect & Liposome & ++ & ++ & ++ & - & $+/-$ \\
\hline JetMessenger & cationic polymer & ++ & ++ & ++ & + & + \\
\hline
\end{tabular}

All the tested formulations could efficiently transfect tumor cells (Figure 1 and summary in Table 1). RiboJuice siRNA was the least efficacious in all cells, whereas RiboJuice mRNA demonstrated the highest transfection efficiency. In splenocytes and PBMCs, RiboJuice mRNA again displayed the best transfection efficacies. The lipoplex JetMessenger and the liposomal MessengerMax also provided robust expression of luciferase in hard-to-transfect primary cell populations. For the purpose of further experiments, we chose MessengerMax, RiboJuice mRNA, and JetMessenger, as they were the most efficient in transfecting mononuclear cells. Our results complement the previous finding that MessengerMax was superior to ScreenFect in transfecting human primary macrophages with ivt mRNA [17].

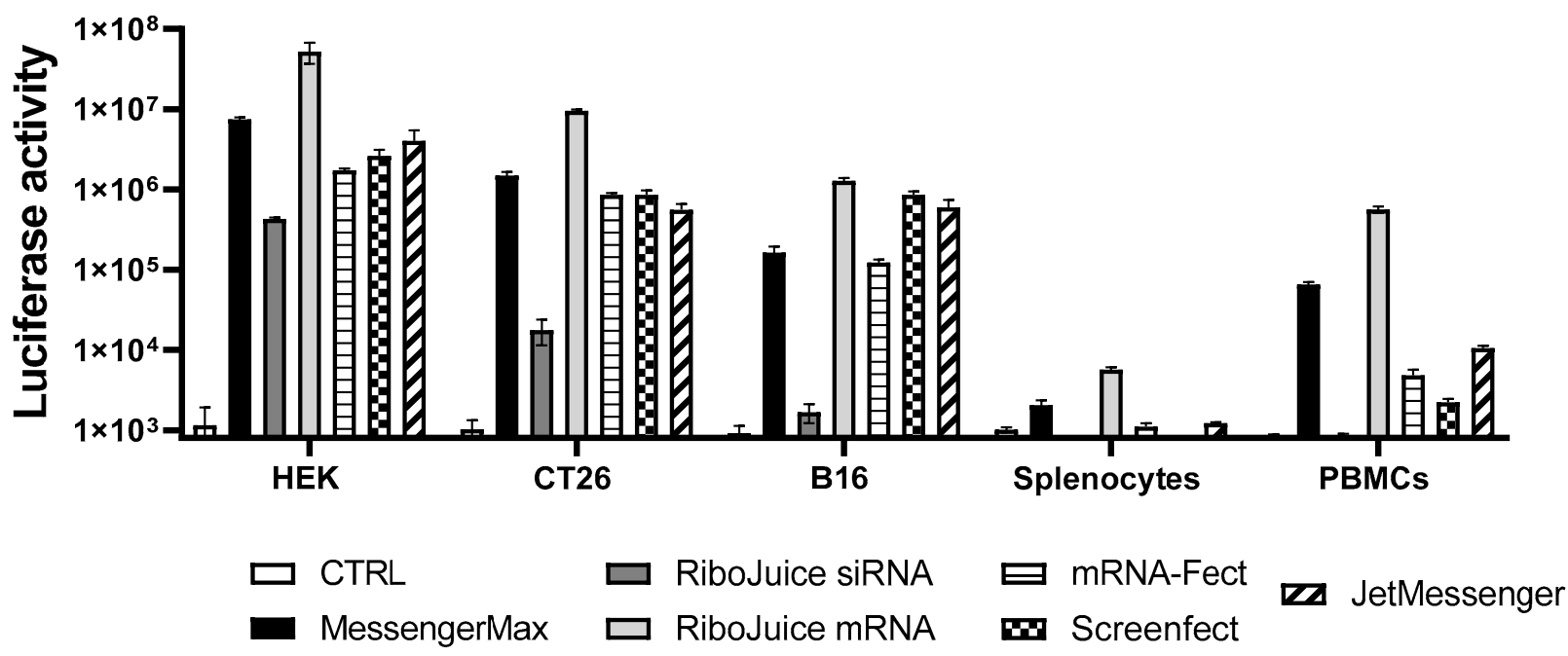

Figure 1. Transfection efficiency of various commercially available transfection reagents. Established human and murine cell lines were transfected with $20 \mathrm{ng}$ mRNA coding for firefly luciferase, whereas primary human PBMCs and murine splenocytes were transfected with $100 \mathrm{ng}$ mRNA. These doses were found as optimal with regard to transfection efficiency and toxicity (Figure S1). Non-transfected cells of each cell type served as the negative control group (CTRL). Luciferase activity was measured $24 \mathrm{~h}$ post transfection in a white 96-well plate. Data represent triplicate mean values; error bars: SD.

\subsection{Effect of the Carrier on the Capacity of mRNA to Stimulate an Innate Immune Response}

We next evaluated the ability of mRNA encapsulated in these chosen carriers to induce an innate immune response, by monitoring levels of interferon alpha (IFN $\alpha$ ). Foreign RNA can stimulate endosomal Toll-like receptors (TLRs) $[18,19]$. When triggered, TLRs induce specific intracellular pathways that result in the expression of cytokines, including anti-viral type I interferons [14]. Unmodified single-stranded RNA (ssRNA) is recognized by human TLR7, expressed by plasmacytoid DCs (the main producers of IFN $\alpha$ ) and human TLR8, and expressed by monocytes (which are capable of producing large amounts of TNF $\alpha$ ) [20]. 
All the nanoparticles containing non-modified mRNA are immunostimulatory in vitro and induce IFN $\alpha$ release in murine and human immune cells (Figure 2A,B). In human cells, MessengerMax prompted the lowest production of IFN $\alpha$, while mRNA-Fect and JetMessenger induced the highest. In murine splenocytes, RiboJuice and MessengerMax induced the lowest stimulation, whereas JetMessenger again induced the highest signal. This was interesting as both RiboJuice and MessengerMax induced the highest luciferase activity in human PBMCs and murine splenocytes (Figure 1), but this did not correlate with the highest innate immune response in these cells. This could be due to the different mechanisms of entry that the formulations illicit, i.e., the polyplex, JetMessenger, mediates cell entry via endocytosis, which could trigger the endosomal TLRs, leading to a greater IFN $\alpha$ response. Indeed, we also used protamine/RNA complexes as a positive control, where the cationic protamine forms nanoparticles with negatively charged RNA. These nanoparticles enter the cells via endosomes and induce elevated levels of IFN $\alpha$ (Figure 2A,B). IFN $\alpha$ stimulation is an important factor in the context of immunomonitoring in vitro, as a strong IFN $\alpha$ response can limit T-cell proliferation [21]; therefore, it may be preferred to prevent the induction of a strong IFN $\alpha$ response in cell cultures.

B
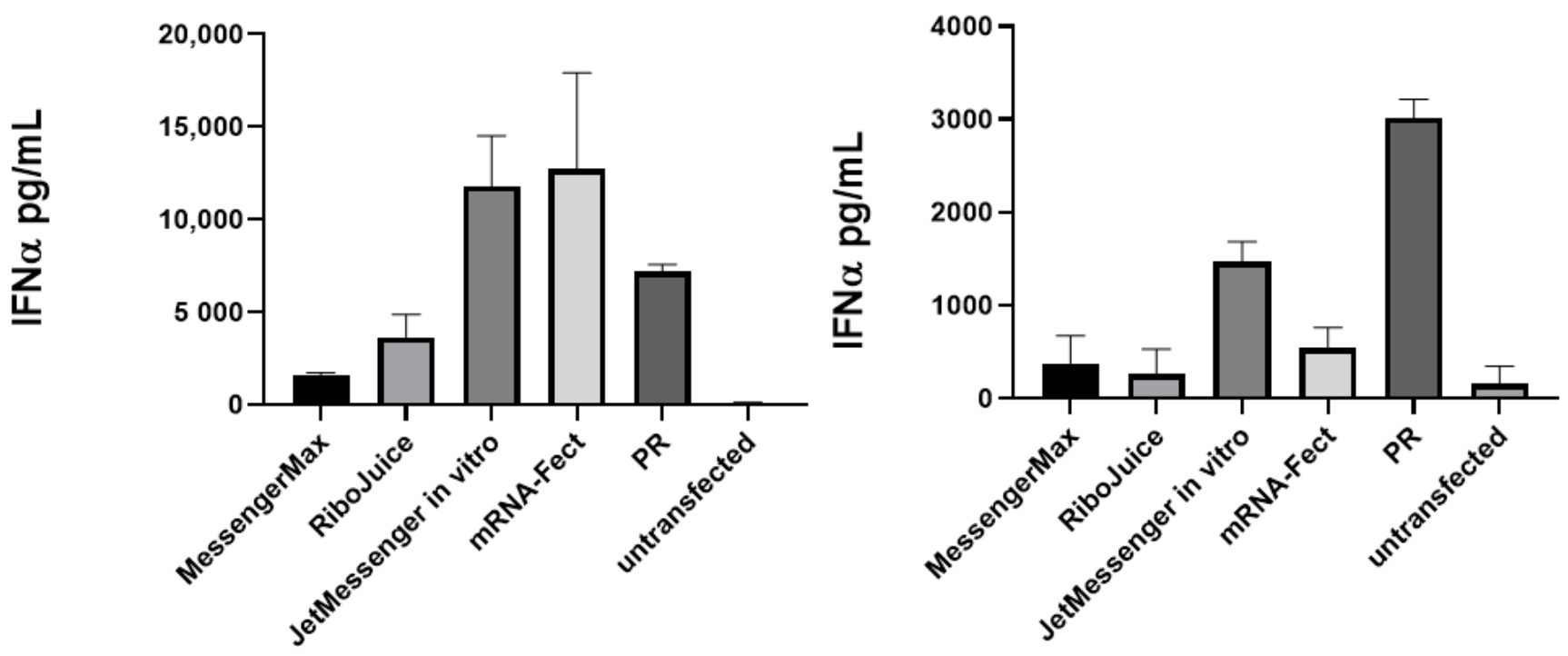

Figure 2. Immune stimulation of mRNA carriers in human PBMCs and murine splenocytes. Cells were seeded at 100,000 cells per well in a 96-well plate and transfected with $200 \mathrm{ng}$ of mRNA per well. Twenty-four hours post transfection supernatants were subjected to ELISA analysis. Selected transfection reagents were tested for their ability to stimulate the production of IFN $\alpha$ in human (A) and murine (B) immune cells. PR: protamine-RNA nanoparticles. Data represent triplicate mean values; error bars: SD.

\subsection{Optimising mRNA-Based T-Cell Immunomonitoring In Vitro in Murine Cells}

To deter production of IFN $\alpha$, next we sought to evaluate the effects of immuno-silent ivt mRNA, where uridine was replaced with pseudouridine (as previously described [22] ). Substitution of uridine with pseudouridine in the ivt mRNA diminished the effects of the formulations to induce expression of IFN $\alpha$ (Figure S2).

To test whether particle-based mRNA transfection in immune cells induces detectable adaptive immune responses, we monitored the secretion of interleukin-2 (IL-2) in splenocytes cultured from OT1 mice. OT1 T cells express the transgenic T-cell receptor that recognizes ovalbumin (OVA) peptide. T cells are stimulated when they recognize the OVA peptide SIINFEKL (residues 257-264) presented on the MHC class I molecule, H-2 Kb [23]. As RiboJuice mRNA, MessengerMax, and JetMessenger gave the highest luciferase expression in PBMCs, they were used to transfect splenocytes from OT1 with ivt mRNA-coding 
ovalbumin. We observed elevated IL-2 levels in cells transfected with all formulations containing ovalbumin-coding mRNA, while particles containing luciferase-coding mRNA did not induce any response in OT1 cells (Figure 3).

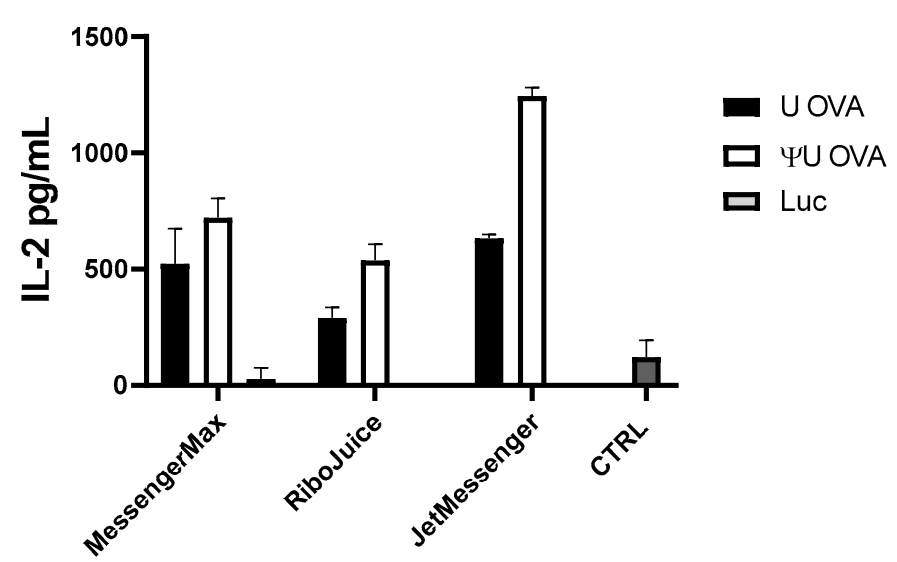

Figure 3. OT1 murine splenocytes were cultivated for $24 \mathrm{~h}$ with ovalbumin-coding (OVA) or luciferase-coding (Luc) mRNA. Ovalbumin-coding mRNA induced an adaptive immune response in OT1 cells, which is indicated by elevated IL-2 levels. Non-transfected OT1 cells served as the negative control group (CTRL). Data represent triplicate mean values; error bars: SD.

Since the induction of type I interferons can limit the proliferation of $\mathrm{T}$ cells and synthesis of new proteins, including cytokines, we compared the impact of substituting immuno-stimulating uridine (U) with immuno-silent pseudouridine ( $\Psi U$ ) on the capacity of ivt mRNAs to activate OT1 splenocytes. Indeed, immuno-silent mRNA induced stronger activation of OT1 T cells than immuno-stimulating ivt mRNA (Figure 3). The greatest improvement was observed with JetMessenger, which is consistent with the high induction of IFN $\alpha$ triggered by this polyplex formulation when it contains unmodified mRNA (Figure 2). Immuno-silent ivt mRNA formulated with MessengerMax had little impact on the production of IL-2 compared to immuno-stimulatory ivt mRNA; this is probably due to the low immuno-stimulatory effects of the MessengerMax formulation, which promotes only slight IFN $\alpha$ expression (Figure 2).

Therefore, we were able to establish optimal ivt mRNA formulations for immunomonitoring that displayed limited innate and augmented adaptive immune responses.

\section{4. mRNA-Based In Vitro Immunomonitoring of Human T-Cells in Response to an Influenza Viral Protein}

We also evaluated our lipofection-based immunomonitoring approach in an ex vivo human system. To that end, PBMCs from an HLA-A*0201 healthy donor were isolated and incubated with formulated mRNAs that encoded the influenza matrix protein M1 (Flu M1) or luciferase as a control. Formulations tested were RiboJuice mRNA and MessengerMax, which induced the greatest expression of luciferase in PBMCs and the lowest induction of IFN $\alpha$ (Figures 1 and 2). IFN $\alpha$ levels were measured in supernatants from all treated groups $24 \mathrm{~h}$ post transfection. IFN $\alpha$ could be strongly induced only in groups cultured with immuno-stimulating ivt mRNA and not in groups cultured with immuno-silent mRNA (Figure S3).

Next, we tested the expansion of Flu M1-specific T cells in PBMCs transfected with immuno-stimulatory or immuno-silent ivt mRNA with MessengerMax and RiboJuice formulations. RiboJuice mRNA was slightly more efficacious than MessengerMax at promoting an adaptive immune response, as determined by tetramer-specific staining for Flu M1 HLA-A*0201-restricted T cells (Figure 4). Furthermore, the immuno-silent ivt mRNA induces an augmented higher percentage of CD8+ T cells compared with immuno-stimulatory ivt mRNA with both RiboJuice and MessengerMax (Figure 4). Meanwhile, the method was tested using frozen PBMCs from healthy HLA-A*0201 donors 
(Figure S4). Again, culturing cells in the presence of mRNA coding the Flu matrix M1 protein formulated in MessengerMax allowed the amplification of T cells specific for the immunodominant Flu M1 HLA-A*0201 epitope.

A

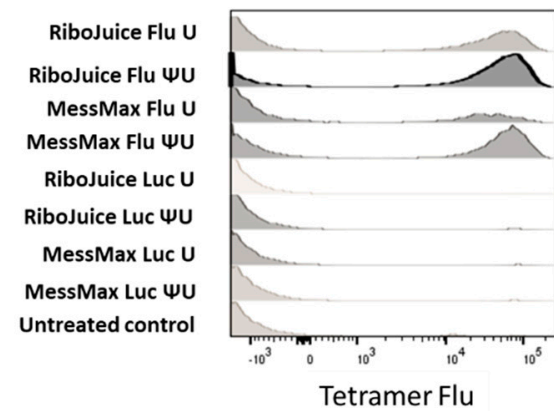

B

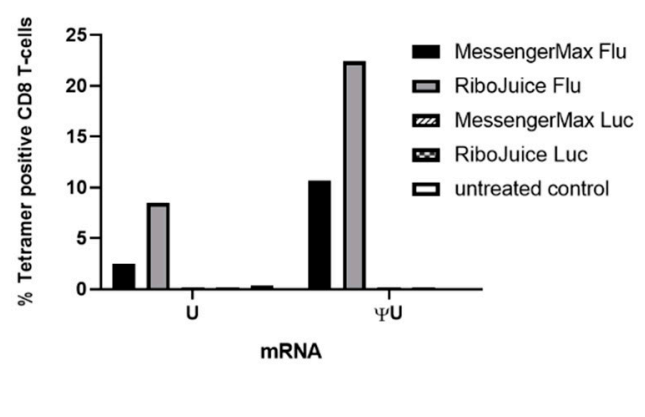

Figure 4. Monitoring of Flu M1-specific immune responses in human PBMCs following transfection with ivt mRNA coding the Flu matrix M1 protein. (A) At day 12 of culture, the cells were stained with antibodies and a fluorescent MHC class I tetramer specific for Flu M1 HLA-A*0201-restricted T cells, and analyzed by FACS. (B) Comparison of the percentage of Flu M1 MC class I tetramer positive T cells after transfection with immuno-stimulating ("U") or immuno-silent (“ $\Psi U$ ") mRNA coding for the Flu M1 HLA-A*0201 epitope.

Collectively, these data show that mRNA-transfected PBMCs efficiently expressed and presented the mRNA-encoded Flu M1 epitope to T cells, and that Flu M1-specific T cells proliferated when the PBMCs were transfected with Flu M1 mRNA, but not when transfected with Luc-coding mRNA. These results were further confirmed in two other HLA-A2-positive donors (Figure S4). Complementing findings from the murine system, stimulation of the adaptive immune response was stronger in vitro with immuno-silent RNA, compared to immuno-stimulating RNA: 22.4 versus $8.52 \%$ for RiboJuice and 10.7 versus $2.49 \%$ for MessengerMax, respectively (Figure 4B). This also corroborates the previous finding that type I interferon prevents proliferation of T cells in vitro [21,24,25].

\section{Discussion}

Synthetic mRNA has been successfully used as a vector for induction of antigenspecific immune responses in vitro and in vivo. Immunomonitoring using mRNA transfection in PBMCs is a fast and easy method compared to mRNA transfection in dendritic cells. Currently, mRNA-based immunomonitoring requires electroporation, which although efficacious hinders the adoption of the method, particularly as it requires special equipment and high amounts of mRNA (usually 10 micrograms per electroporation). We demonstrate here that newly available efficacious reagents, i.e., highly functional stabilized mRNA and efficacious transfection reagents, allow us to transfect human primary blood cells and murine splenocytes (Figure 1). We aim to develop mRNA transfection via nanoparticles in PBMCs into a quick and reliable technique for monitoring the antigen-specific T-cell responses present in periphery. With such an approach, we aimed to optimize and achieve a simple and robust method, easily accessible to every laboratory without the need for specialized equipment or excessive time consumption and additionally, with a lower amount of mRNA required (1 microgram per $\mathrm{mL}$ ).

Among all tested transfection reagents, we found that transfection efficiency depends on cell origin and cell type, and there is no single reagent that would be optimal for all cell types (Table 1 and Figure S5). Although RiboJuice mRNA appears to be globally the most efficacious reagent, it is less stable and has to be used within 5 min of formulation, making it slightly less easy to use than others, including MessengerMax, which is an efficacious, stable, and robust transfection reagent, as it could efficiently deliver mRNA to all tested cell types. During the course of experimentation, we optimized conditions that led to detectable expression levels of the protein encoded by exogenous mRNA in primary mouse 
and human immune cells. In addition, such mRNA-transfected cells presented MHC class I epitopes to T cells, as documented by the specific activation of SIINFEKL specific mouse $\mathrm{T}$-cells or specific expansion of influenza-specific human $\mathrm{T}$ cells upon transfection with antigen-encoding mRNA.

We observed quantitative differences in cytokine release depending on the choice of type of mRNA used in transfection. Unmodified mRNA induced a strong innate immune response in primary cells in all tested conditions; in human PBMCs and murine splenocytes. Meanwhile, mRNA with pseudouridine in its sequence in place of uridine, so-called "immuno-silent" mRNA, did not induce innate immune responses, but better prompted an adaptive immune response, i.e., elevated IL-2 levels in mouse OT1 cells transfected with ovalbumin-coding mRNA (Figure 3) and increased proliferation of human epitope-specific T cells (Figure 4A and Figure S4). It is well established that RNA activates cells of the innate immune system by stimulating Toll-like receptors, specifically TLR3, TLR7, and TLR8 $[26,27]$. However, when naturally occurring modified nucleosides, for example, pseudouridine, are incorporated into the transcript, the TLRs can no longer be triggered [22]. Studies revealed that incorporating pseudouridine into mRNA not only suppresses RNA-mediated immune activation in vitro and in vivo, but also enhances the translational capacity of the RNA [26]. One cause of this translational difference is that pseudouridine-containing mRNA activates RNA-dependent protein kinase (PKR) less efficiently than uridine-containing mRNA [28]. Another likely contributing factor to the enhanced translation observed with pseudouridine modification is an increase in biological stability of the mRNAs [26]. Indeed, higher resistance to hydrolysis by phosphodiesterases from snake venom and spleen was reported when uridine was replaced with pseudouridine in dinucleotide substrates [29]. Other studies have also demonstrated that pseudouridine stabilizes RNA secondary structures by promoting base stacking, which could slow degradation [30].

Immunomonitoring has become increasingly relevant in the many medical fields. In immuno-oncology it is used for the identification of potential prognostic or predictive immune biomarkers and a better understanding of their underlying mechanisms of action, leading to improved personalized treatments [31]. In addition, the SARS-CoV-2 pandemic that emerged in late 2019 revealed the necessity of a rapid and simple immunomonitoring method. Development of new therapies as well as new pathogen-specific vaccines and treatments require proper assessment of innate and adaptive immune responses, as human immune systems are highly variable [32]. A number of researchers are studying the immune response to SARS-CoV-2 to reveal a systems-level perspective on the immune system changes during the acute and recovery phases of severe COVID-19 disease [33]. We envision that our simplified method for immunomonitoring presented here will be widely implemented to study the T-cell immune responses triggered in patients suffering from infections, cancers, and autoimmunity or inflammatory diseases.

Supplementary Materials: The following are available online at https:/ / www.mdpi.com/article / 10.3390/v13071232/s1, Figure S1: mRNA dose-dependent (A) transfection efficacy and (B) toxicity in human PBMCs and HEK cells; Figure S2: Differences in innate immune response in murine splenocytes transfected with mRNA containing uridine (U) or pseudouridine ( $\Psi U)$ in various carriers; Figure S3: IFN $\alpha$ levels in supernatants from hPBMCs $24 \mathrm{~h}$ post transfection with indicated mRNA containing uridine $(\mathrm{U})$ or pseudo-uridine $(\Psi \mathrm{U})$ in various carriers and the negative control group (CTRL); Figure S4: Monitoring of Flu M1-specific immune responses in human PBMCs following transfection with ivt mRNA coding the Flu matrix M1 protein (Flu) or Luciferase (Luc); Figure S5: Flow cytometry analysis of hPBMCs transfected with different reagents containing ZsGreen-coding mRNA.

Author Contributions: Conceptualization, N.T.J., J.F., and S.P.; methodology, N.T.J., J.F., and S.P.; validation, N.T.J., J.F., S.P., and M.M.; formal analysis, N.T.J., J.F., S.P., and M.M.; investigation, N.T.J., J.F., C.G., and S.P.; resources, S.L., L.E.F., E.G., T.M.K., and S.P.; writing-original draft preparation, N.T.J., M.M., and S.P.; writing—review and editing, N.T.J., J.F., S.L., L.E.F., E.G., T.M.K., C.G., M.M., and S.P.; funding acquisition, S.L., L.E.F., E.G., T.M.K., and M.M. All authors have read and agreed to the published version of the manuscript. 
Funding: This research was funded by the University of Zurich (URPP "Translational Cancer Research), the department of Dermatology at the University Hospital of Zurich, the "Stiftung für wissenschaftliche Forschung an der Universität Zürich", the Swiss National Science Found NRP 78 program, grant number 4078PO_198321 and the EU grant NEWmRNA (Horizon 2020 research and innovation programme No. 965135).

Institutional Review Board Statement: The study was conducted according to the guidelines of the Declaration of Helsinki, and all animal experiments were performed in accordance with the regulations and guidelines of and with ethical approval from the Cantonal Veterinary Office of Zürich, Switzerland.

Informed Consent Statement: Not applicable.

Data Availability Statement: Data is contained within the article.

Acknowledgments: We thank Pål Johansen for providing us with OT1 mice.

Conflicts of Interest: The authors declare no conflict of interest.

\section{References}

1. Duffy, D. Standardized immunomonitoring: Separating the signals from the noise. Trends Biotechnol. 2018, 36, 1107-1115. [CrossRef]

2. Hartmann, F.J.; Babdor, J.; Gherardini, P.F.; Amir, E.-A.D.; Jones, K.; Sahaf, B.; Marquez, D.M.; Krutzik, P.; O’Donnell, E.; Sigal, N.; et al. Comprehensive immune monitoring of clinical trials to advance human immunotherapy. Cell Rep. 2019, 28, 819-831.e4. [CrossRef]

3. Teufel, R.; Carralot, J.P.; Scheel, B.; Probst, J.; Walter, S.; Jung, G.; Hoerr, I.; Rammensee, H.G.; Pascolo, S. Human peripheral blood mononuclear cells transfected with messenger rna stimulate antigen-specific cytotoxic t-lymphocytes in vitro. Cell. Mol. Life Sci. CMLS 2005, 62, 1755-1762. [CrossRef] [PubMed]

4. Uenaka, A.; Wada, H.; Isobe, M.; Saika, T.; Tsuji, K.; Sato, E.; Sato, S.; Noguchi, Y.; Kawabata, R.; Yasuda, T.; et al. T cell immunomonitoring and tumor responses in patients immunized with a complex of cholesterol-bearing hydrophobized pullulan (chp) and ny-eso-1 protein. Cancer Immun. Arch. 2007, 7, 9.

5. Medaglini, D.; Siegrist, C.-A. Immunomonitoring of human responses to the rvsv-zebov ebola vaccine. Curr. Opin. Virol. 2017, 23, 88-94. [CrossRef] [PubMed]

6. Knights, A.J.; Nuber, N.; Thomson, C.W.; de la Rosa, O.; Jäger, E.; Tiercy, J.M.; van den Broek, M.; Pascolo, S.; Knuth, A.; Zippelius, A. Modified tumour antigen-encoding mrna facilitates the analysis of naturally occurring and vaccine-induced cd4 and cd8 $\mathrm{t}$ cells in cancer patients. Cancer Immunol. Immunother. 2009, 58, 325-338. [CrossRef]

7. Bins, A.D.; Jorritsma, A.; Wolkers, M.C.; Hung, C.-F.; Wu, T.C.; Schumacher, T.N.M.; Haanen, J.B.A.G. A rapid and potent DNA vaccination strategy defined by in vivo monitoring of antigen expression. Nat. Med. 2005, 11, 899-904. [CrossRef] [PubMed]

8. Mulligan, M.J.; Lyke, K.E.; Kitchin, N.; Absalon, J.; Gurtman, A.; Lockhart, S.; Neuzil, K.; Raabe, V.; Bailey, R.; Swanson, K.A.; et al. Phase i/ii study of covid-19 rna vaccine bnt162b1 in adults. Nature 2020, 586, 589-593. [CrossRef]

9. Kreiter, S.; Selmi, A.; Diken, M.; Sebastian, M.; Osterloh, P.; Schild, H.; Huber, C.; Türeci, O.; Sahin, U. Increased antigen presentation efficiency by coupling antigens to mhc class i trafficking signals. J. Immunol. 2008, 180, 309-318. [CrossRef] [PubMed]

10. Van Camp, K.; Cools, N.; Stein, B.; Van de Velde, A.; Goossens, H.; Berneman, Z.N.; Van Tendeloo, V. Efficient mrna electroporation of peripheral blood mononuclear cells to detect memory t cell responses for immunomonitoring purposes. J. Immunol. Methods 2010, 354, 1-10. [CrossRef]

11. Hiura, M.; Ueno, K.; Suehiro, Y.; Hazama, S.; Oka, M.; Imai, K.; Hinoda, Y. A simple immunomonitoring procedure for mrna-loaded dendritic cell therapy. Tumor Biol. 2007, 28, 350-357. [CrossRef]

12. Tusup, M.; Kundig, T.; Pascolo, S. An eif4g-recruiting aptamer increases the functionality of in vitro transcribed mrna. EPH Int. J. Med. Health Sci. 2018, 4, 29-35.

13. Danckwardt, S.; Hentze, M.W.; Kulozik, A.E. $3^{\prime}$ end mrna processing: Molecular mechanisms and implications for health and disease. EMBO J. 2008, 27, 482-498. [CrossRef]

14. Tusup, M.; Pascolo, S. Generation of immunostimulating $130 \mathrm{~nm}$ protamine-rna nanoparticles. In Rna Vaccines: Methods and Protocols; Kramps, T., Elbers, K., Eds.; Springer: New York, NY, USA, 2017; pp. 155-163.

15. Jarzebska, N.T.; Lauchli, S.; Iselin, C.; French, L.E.; Johansen, P.; Guenova, E.; Kündig, T.M.; Pascolo, S. Functional differences between protamine preparations for the transfection of mrna. Drug Deliv. 2020, 27, 1231-1235. [CrossRef] [PubMed]

16. Gotch, F.; Rothbard, J.; Howland, K.; Townsend, A.; McMichael, A. Cytotoxic t lymphocytes recognize a fragment of influenza virus matrix protein in association with hla-a2. Nature 1987, 326, 881-882. [CrossRef]

17. Moradian, H.; Roch, T.; Lendlein, A.; Gossen, M. Mrna transfection-induced activation of primary human monocytes and macrophages: Dependence on carrier system and nucleotide modification. Sci. Rep. 2020, 10, 4181. [CrossRef]

18. Scheel, B.; Braedel, S.; Probst, J.; Carralot, J.-P.; Wagner, H.; Schild, H.; Jung, G.; Rammensee, H.-G.; Pascolo, S. Immunostimulating capacities of stabilized rna molecules. Eur. J. Immunol. 2004, 34, 537-547. [CrossRef] [PubMed]

19. Scheel, B.; Aulwurm, S.; Probst, J.; Stitz, L.; Hoerr, I.; Rammensee, H.-G.; Weller, M.; Pascolo, S. Therapeutic anti-tumor immunity triggered by injections of immunostimulating single-stranded rna. Eur. J. Immunol. 2006, 36, 2807-2816. [CrossRef] 
20. Jarrossay, D.; Napolitani, G.; Colonna, M.; Sallusto, F.; Lanzavecchia, A. Specialization and complementarity in microbial molecule recognition by human myeloid and plasmacytoid dendritic cells. Eur. J. Immunol 2001, 31, 3388-3393. [CrossRef]

21. Erickson, S.; Sangfelt, O.; Castro, J.; Heyman, M.; Einhorn, S.; Grandér, D. Interferon-alpha inhibits proliferation in human $t$ lymphocytes by abrogation of interleukin 2-induced changes in cell cycle-regulatory proteins. Cell Growth Differ. Mol. Biol. J. Am. Assoc. Cancer Res. 1999, 10, 575-582.

22. Karikó, K.; Buckstein, M.; Ni, H.; Weissman, D. Suppression of rna recognition by toll-like receptors: The impact of nucleoside modification and the evolutionary origin of rna. Immunity 2005, 23, 165-175. [CrossRef]

23. Clarke, S.R.; Barnden, M.; Kurts, C.; Carbone, F.R.; Miller, J.F.; Heath, W.R. Characterization of the ovalbumin-specific tcr transgenic line ot-i: Mhc elements for positive and negative selection. Immunol. Cell Biol. 2000, 78, 110-117. [CrossRef] [PubMed]

24. Marrack, P.; Kappler, J.; Mitchell, T. Type i interferons keep activated t cells alive. J. Exp. Med. 1999, 189, 521-530. [CrossRef]

25. Dondi, E.; Rogge, L.; Lutfalla, G.; Uzé, G.; Pellegrini, S. Down-modulation of responses to type i ifn upon $t$ cell activation. J. Immunol. 2003, 170, 749-756. [CrossRef] [PubMed]

26. Karikó, K.; Muramatsu, H.; Welsh, F.A.; Ludwig, J.; Kato, H.; Akira, S.; Weissman, D. Incorporation of pseudouridine into mrna yields superior nonimmunogenic vector with increased translational capacity and biological stability. Mol. Ther. 2008, 16, 1833-1840. [CrossRef]

27. Heil, F.; Hemmi, H.; Hochrein, H.; Ampenberger, F.; Kirschning, C.; Akira, S.; Lipford, G.; Wagner, H.; Bauer, S. Species-specific recognition of single-stranded rna via toll-like receptor 7 and 8. Science 2004, 303, 1526. [CrossRef]

28. Anderson, B.R.; Muramatsu, H.; Nallagatla, S.R.; Bevilacqua, P.C.; Sansing, L.H.; Weissman, D.; Karikó, K. Incorporation of pseudouridine into mrna enhances translation by diminishing pkr activation. Nucleic Acids Res. 2010, 38, 5884-5892. [CrossRef] [PubMed]

29. Naylor, R.; Ho, N.W.; Gilham, P.T. Selective chemical modifications of uridine and pseudouridine in polynucleotides and their effect on the specificities of ribonuclease and phosphodiesterases. J. Am. Chem. Soc. 1965, 87, 4209-4210. [CrossRef]

30. Davis, D.R. Stabilization of rna stacking by pseudouridine. Nucleic Acids Res 1995, 23, 5020-5026. [CrossRef] [PubMed]

31. Macchia, I.; La Sorsa, V.; Ruspantini, I.; Sanchez, M.; Tirelli, V.; Carollo, M.; Fedele, G.; Leone, P.; Schiavoni, G.; Buccione, C.; et al. Multicentre harmonisation of a six-colour flow cytometry panel for naïve/memory t cell immunomonitoring. J. Immunol. Res. 2020, 2020, 1938704. [CrossRef]

32. Brodin, P.; Davis, M.M. Human immune system variation. Nat. Rev. Immunol. 2017, 17, 21-29. [CrossRef] [PubMed]

33. Rodriguez, L.; Pekkarinen, P.T.; Lakshmikanth, T.; Tan, Z.; Consiglio, C.R.; Pou, C.; Chen, Y.; Mugabo, C.H.; Nguyen, N.A.; Nowlan, K.; et al. Systems-level immunomonitoring from acute to recovery phase of severe covid-19. Cell Rep. Med. 2020, 1, 100078. [CrossRef] [PubMed] 